TPI-MINN-99/02-T

UMN-TH-1738/99

NYU-TH-99/1/01

hep-th/9901111

\title{
Surviving on the Slope: Supersymmetric Vacuum in the Theories Where It Is Not Supposed to Be
}

\author{
G. Dvali \\ Physics Department, New York University, New York, NY 10003 \\ and \\ International Center for Theoretical Physics, Trieste, I-34014, Italy \\ and \\ M. Shifman \\ Theoretical Physics Institute, University of Minnesota, Minneapolis, MN 55455
}

\begin{abstract}
In supersymmetric models with the run-away vacua or with the stable but nonsupersymmetric ground state there exist stable field configurations (vacua) which restore one half of supersymmetry and are characterized by constant positive energy density. The formal foundation for such vacua is provided by the central extension of the $\mathcal{N}=1$ superalgebra with the infinite central charge.
\end{abstract}


In Ref. [1] we found a class of unconventional solutions, which exist in supersymmetric theories with a vacuum moduli space, and are characterized by (i) constant energy density; (ii) topological stability. They can be considered as a limiting case of the domain walls (sometimes we deal with the so-called constant phase configurations, sometimes with the winding phase configurations, see Sec. 5 of [1]). One half of supersymmetry may or may not be preserved on these solutions. Because of their topological stability they can become vacua of a theory breaking a part of the Lorentz invariance and supersymmetry. Thus, this is a particular realization of the dynamical compactification, the idea central in Ref. [1].

The existence of the topologically stable vacua with the purely gradient (constant) energy density is intuitively clear in the examples considered in Ref. [四] since in these examples there exist moduli forming a continuous manifold of supersymmetric vacuum states. Being physically inequivalent, the vacua are degenerate, the vacuum energy density vanishes. The vacuum moduli spaces occur frequently in supersymmetric theories.

Here we discuss another class of supersymmetric theories, which, within the standard understanding, have no supersymmetric vacua at all: either there is no vacuum whatsoever (the scalar potential has a "run-away" behavior), or the vacuum state exists but is non-supersymmetric. Our solutions restore a part of supersymmetry. This pattern is quite unusual - normally, stable field configurations with higher energy density have less supersymmetry than the ground state. Our analysis shows that the opposite situation is also possible.

First, we will consider the so-called run-away theories. The most well-known example of this type is $\mathrm{SU}(2) \mathrm{SQCD}$ with one massless flavor [2] (in general, $\mathrm{SU}(N)$ SQCD with $N-1$ flavors). At any finite values of fields the minimal energy is not achieved. One approaches the vanishing energy density at infinitely distant points in the space of fields. So, there is no vacuum in the conventional sense of this word. Then, we will consider models with the spontaneous breaking of supersymmetry of the O'Raifeartaigh type [3]. Both phenomena are quite common in the zoo of supersymmetric theories. The O'Raifeartaigh models appear as a low-energy limit of various gauge models producing the dynamical supersymmetry breaking (for a recent review see [4]).

We will show that in both cases BPS saturated solutions exist; they are stable under all localized perturbations, preserve one half of the original supersymmetry and, thus, present supersymmetric vacuum states. It may well happen that such solutions in the future will become a component of a phenomenologically successful scenario (e.g. [5]).

Let us start from the run-away vacua. Many models with the run-away vacuum were considered in the literature. For definitness we focus on models with the logarithmic superpotential for the moduli,

$$
\mathcal{L}=\frac{1}{4} \int d^{2} \theta d^{2} \bar{\theta} \bar{\Phi} \Phi+\left(\frac{1}{2} \int d^{2} \theta \mathcal{W}+\text { H.c }\right)
$$


where

$$
\mathcal{W}=-i M^{3} \ln \Phi .
$$

The parameter $M$ can be always chosen to be real. The scalar potential $V$ is proportional to $|\phi|^{-2}$ (a "mountain peak" centered at the origin in the space of fields). The run-away behavior is obvious.

For the wall-like solutions (i.e. static field configurations depending only on one coordinate $z$ ) the condition of the BPS saturation takes the form [1]

$$
\frac{\partial \phi}{\partial z}=\frac{\partial \overline{\mathcal{W}}}{\partial \bar{\phi}} .
$$

It is quite obvious that, given the superpotential (2), the solution of Eq. (3) of the winding-phase type is

$$
\phi_{0}(z)=m e^{i \alpha(z)}, \quad \alpha(z)=\frac{M^{3}}{m^{2}} z .
$$

For convenience we assumed $m$ to be real; its absolute value is arbitrary (one can always pass to a complex $m$ by a phase rotation of $\Phi)$. The solution (3) preserves two out of four supercharges. The energy functional can be written as

$$
E=\int d^{3} x\left\{\left|\frac{\partial \phi}{\partial z}+i \frac{M^{3}}{\bar{\phi}}\right|^{2}+\left|\frac{\partial \phi}{\partial x}\right|^{2}+\left|\frac{\partial \phi}{\partial y}\right|^{2}+\left(\frac{\partial \mathcal{W}}{\partial z}+\text { H.c. }\right)\right\} .
$$

The corresponding vacuum energy density is

$$
\mathcal{E}=2 \frac{M^{6}}{m^{2}} .
$$

Equation (5) explicitly demonstrates that the system is stable under spatially localized perturbations. Indeed, if $\phi=\phi_{0}+\delta \phi$, and $\delta \phi$ vanishes at infinity,

$$
\delta E=\int d^{3} x\left\{\left|\frac{\partial(\delta \phi)}{\partial z}-i \frac{M^{3}}{\bar{\phi}^{2}} \delta \bar{\phi}\right|^{2}+\left|\frac{\partial(\delta \phi)}{\partial x}\right|^{2}+\left|\frac{\partial(\delta \phi)}{\partial y}\right|^{2}\right\} .
$$

There are no negative modes. Thus, we get a continuous family of vacua with a constant energy density labeled by the parameter $m$.

Another way to understand the stability is by compactifying the coordinate $z$ on a circle of the radius $R$ and then taking the limit $R \rightarrow \infty$. For finite $R$ only a discrete number of solutions is allowed $M^{3} / m^{2}=n / R \quad(n=1,2 .$.$) . Thus, M^{3} / m^{2}$ is a topologically conserved winding number density which guaranties the stability of the configuration. Now, taking the limit $R, n \rightarrow \infty$ with $n / R$ fixed we recover Eq. (4).

Now, let us discuss a model presenting a classic example of the spontaneous supersymmetry breaking (the O'Raifeartaigh mechanism [3]). It includes three chiral superfields, $\Phi_{1,2,3}$, with the superpotential

$$
\mathcal{W}=\lambda_{1} \Phi_{1}\left(\Phi_{3}^{2}-M^{2}\right)+\mu \Phi_{2} \Phi_{3} .
$$


Again, it is convenient to choose the parameters $\lambda_{1}, M$ and $\mu$ real. Superpotential of the type (8) appear in the low-energy limit of various gauge field theories with matter. If $M^{2}<\mu^{2} /\left(2 \lambda_{1}^{2}\right)$ the minimum of energy is achieved at $\phi_{2}=\phi_{3}=0$ and $\phi_{1}$ undetermined. At the minimum the $F_{1}$ term does not vanish, $F_{1}=\lambda_{1} M^{2}$, so that supersymmetry is broken, and the vacuum energy density $\mathcal{E}=\lambda_{1}^{2} M^{4}$. Note that the flat direction along $\phi_{1}$ is lifted by the $Z$ factor arising as a (perturbative) quantum correction to the kinetic term.

Thus, in the flat vacuum SUSY is totally broken. Instead, one can try to find a BPS saturated wall-like solution preserving one half of SUSY. The BPS saturation conditions now take the form

$$
\frac{\partial \phi_{i}}{\partial z}=\frac{\partial \overline{\mathcal{W}}}{\partial \bar{\phi}_{i}}, \quad i=1,2,3 .
$$

They have an obvious solution

$$
\phi_{1}=-\lambda_{1} M^{2} z, \quad \phi_{2}=0, \quad \phi_{3}=0 .
$$

The vacuum energy density for this field configuration is

$$
\mathcal{E}=2 \lambda_{1}^{2} M^{4}
$$

i.e. twice higher than in the Lorentz-invariant non-supersymmetric vacuum. Nevertheless, the configuration (10) is absolutely stable under all localized deformations, much in the same way as in the case of the winding phase configuration of the previous example.

In both cases the residual one half of SUSY guarantees that the fermion-boson degeneracy persists for the excitation modes in the given backgrounds. In the latter case, Eq. (10), the excitation modes from $\Phi_{3}$ are localized in the $z$ direction.

The total vacuum energy gets no quantum corrections due to the BPS-saturated nature of the wall-like solutions considered. However, the $z$-independence of the vacuum energy density is lifted, generally speaking, by quantum corrections to the kinetic term. In the weak coupling regime these quantum corrections are small, however.

The mathematical foundation for the existence of the spatially delocalized vacuum configurations with the residual supersymmetry and a (classically) constant energy density, which we present here, is the central extension of the $\mathcal{N}=1$ superalgebra with an infinite central charge,

$$
\left\{Q_{\alpha} Q_{\beta}\right\}=\Sigma_{\alpha \beta} \mathcal{Z}
$$

where $\Sigma_{\alpha \beta}$ is proportional to the area tensor in the plane perpendicular to the $z$ direction, and

$$
\mathcal{Z}=2\{\mathcal{W}(z=L)-\mathcal{W}(z=-L)\} \propto \text { const } L \rightarrow \infty
$$


in both models considered. This is a natural generalization of the central extensions of the $\mathcal{N}=1$ superalgebra with a finite value of the central charge found and discussed previously [6]. (Note that when we speak of the finite/infinite central charge we do not include in $\mathcal{Z}$ a trivial area factor $\Sigma_{\alpha \beta} \propto A$, which is, of course, infinite since the wall area $A \rightarrow \infty$. For a recent discussion of a general theory of the tensorial central charges in various superalgebras in three and four dimensions see [7].) In the examples discussed in [6] the walls interpolate between a discrete set of vacua related to each other by phase transformations. Therefore, the central charge can take one of several possible (finite) values from a discrete finite set. Whereas in the present case there exists a symmetry of the model per se, or of the vacuum state, under which the superpotential $\mathcal{W}$ gets a shift. This explains why the central charge is infinite.

In the non-supersymmetric context stable soliton-like vacua in the theories without the Lorentz-invariant vacua were discussed in Ref. [8] and, more recently, in Ref. [9], where the question was raised as to the relevance of such configurations in the cosmological setting. In supersymmetric world the models with no supersymmetric vacuum are abundant. The vacua of the type we discuss here may play an important role in the description of the cosmology emerging, in particular, in the context of the TeV Planck scale scenario [10]. First ideas in this direction will be presented in Ref. [5].

Acknowledgments:

This work was supported in part by DOE under the grant number DE-FG0294ER40823.

\section{References}

[1] G. Dvali and M. Shifman, Nucl. Phys. B504 (1997) 127.

[2] I. Affleck, M. Dine, and N. Seiberg, Nucl. Phys. B256 (1985) 557.

[3] L. O’Raifeartaigh, Nucl. Phys. B96 (1975) 331.

[4] E. Poppitz and S. Trivedi, Dynamical Supersymmetry Breaking, hepth/9803107.

[5] G. Dvali and M. Shifman, to be published.

[6] G. Dvali and M. Shifman, Phys. Lett. B396 (1997) 64; (E) , B407 (1997) 452; A. Kovner, M. Shifman, and A. Smilga, Phys. Rev. D56 (1997) 7978; B. Chibisov and M. Shifman, Phys. Rev. D56 (1997) 7990.

[7] S. Ferrara and M. Porrati, Phys. Lett. B423 (1998) 255. 
[8] E. D'Hoker and R. Jackiw, Phys. Rev. D26 (1982) 3517; Phys. Rev. Lett. 50 (1983) 1719; E. D'Hoker, D. Freedman, and R. Jackiw, Phys. Rev. D28 (1983) 2583.

[9] I. Cho and A. Vilenkin, hep-th/9808090.

[10] N. Arkani-Hamed, S. Dimopoulos, and G. Dvali, Phys. Lett. B429 (1998) 263; I. Antoniadis, N. Arkani-Hamed, S. Dimopoulos, and G. Dvali, Phys. Lett. B436 (1998) 257. 